\title{
Prediction of Depression Based on Dysfunctional Attitudes, Personality Traits, and Family Communication Patterns among Patients with Epilepsy
}

\author{
Omsalmeh Mousazadeh, Kobra Haji Alizadeh* \\ Department of Psychology, Bandar Abbas Branch, Islamic Azad University, Bandar Abbas, Iran
}

\section{A BSTRACT}

Introduction: Patients with epilepsy are markedly at the risk of depression. It is important to find out the causes of depression in these patients in order to provide comprehensive health care services. Therefore, this study was done to predict the propensity to the depression based on dysfunctional attitudes, personality traits, and family communication patterns among epileptic patients. Materials and Methods: The present research was a descriptive-correlational study. The population included all patients with epilepsy who referred to the hospitals and medical clinic of Bandar Abbas during the year 2015 from which 150 patients were selected using convenience sampling method. Beck depression Inventory, NEO Five-Factor Inventory, Dysfunctional Attitudes Scale, and Family Communication Patterns Questionnaire were used for data collection. Results: Personality traits of neuroticism, extraversion, agreeableness, and openness as well as communication pattern of conformity can explain together $\% 59.2$ of depression variance in these patients. However, conscientiousness personality trait, dysfunctional attitudes, and communication pattern of conversation cannot predict depression. Conclusion: According to the findings of this study, it can be suggested that training and intervention on improving personality traits and family communication patterns are effective methods to decreasing depression of patients with epilepsy.

Key words:

1. Depression

2. Epilepsy

3. Patients

*Corresponding Author: Kobra Haji Alizadeh

E-mail:ph_alizadeh@yahoo.com 


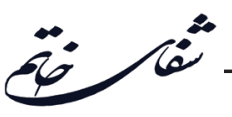

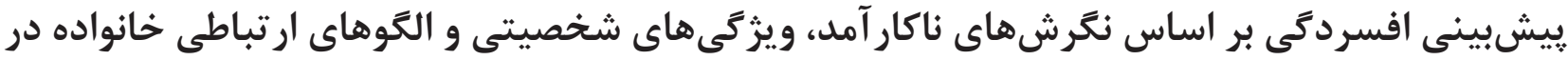

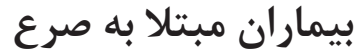

\author{
امسلمه موسىزاده، كبرى حاجى عليزاده" \\ كروه روانشناسى، واحد بندرعباس، دانشكاه آزاد اسلامى، بندرعباس، ايران
}

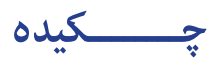

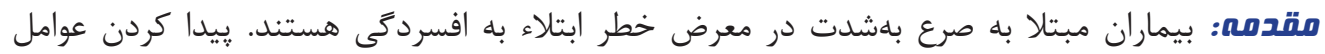

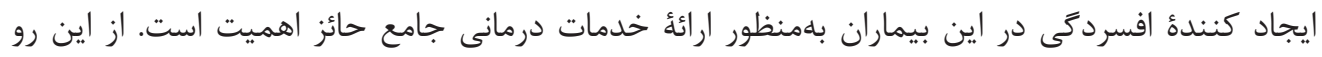

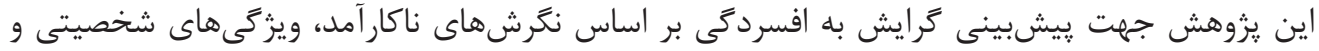

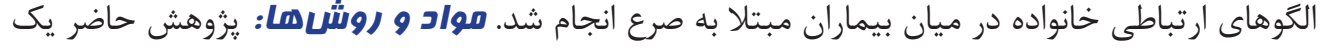

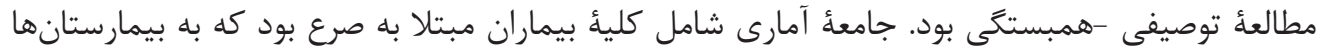

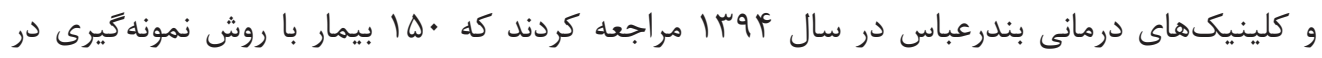

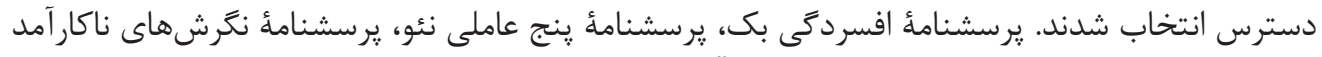

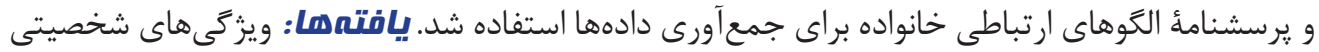

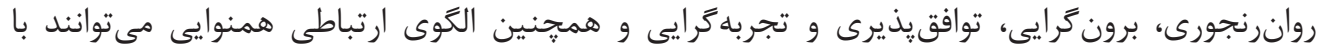

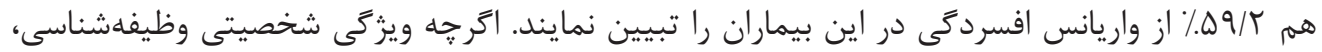

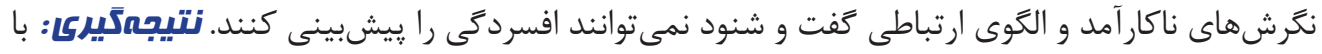

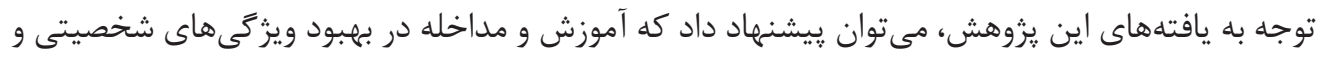

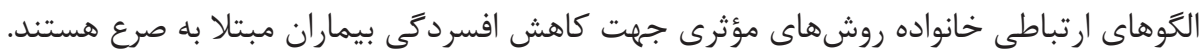

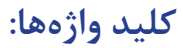

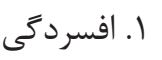
r. r.

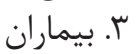




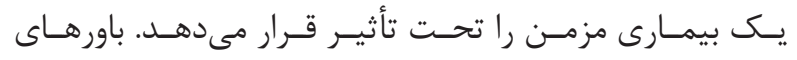

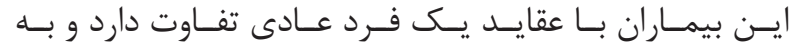

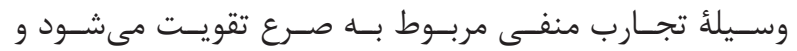

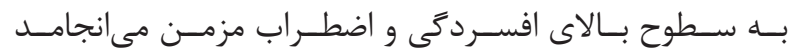

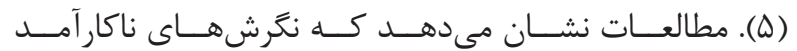

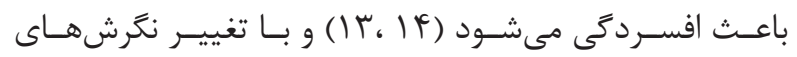

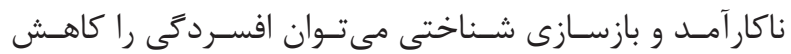

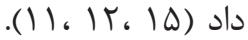

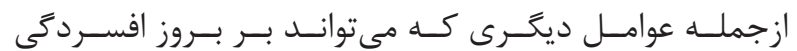

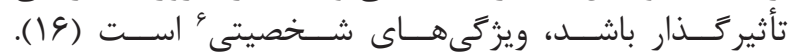

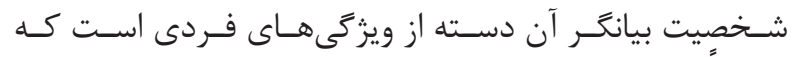

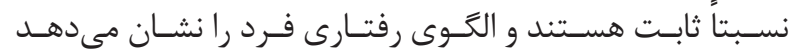

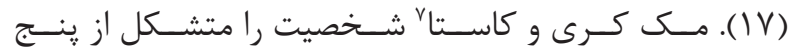

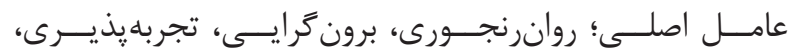

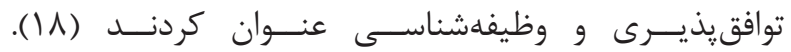

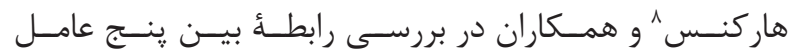

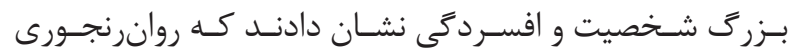

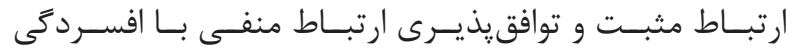

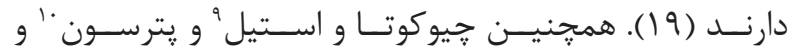

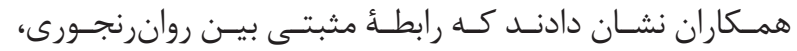

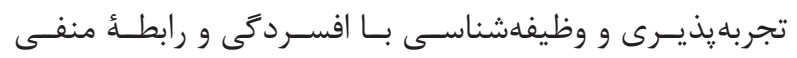

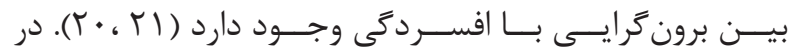

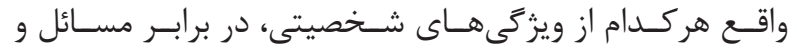

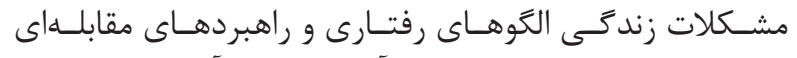

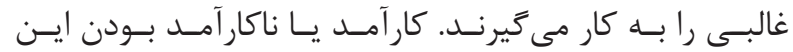

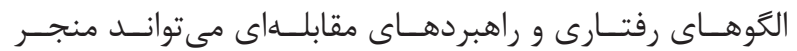

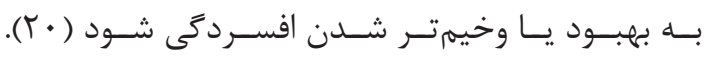

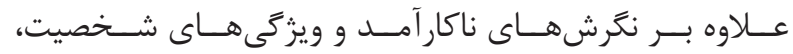

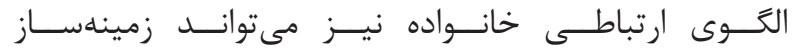

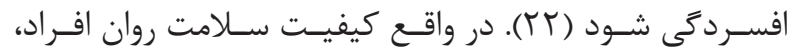

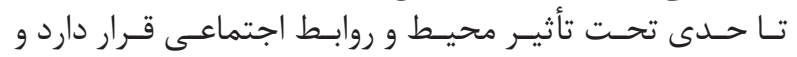

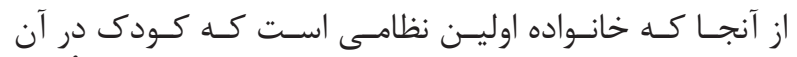

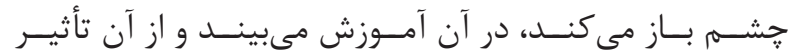

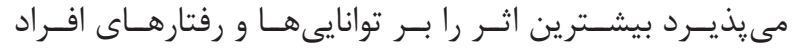

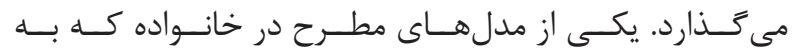

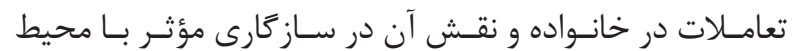

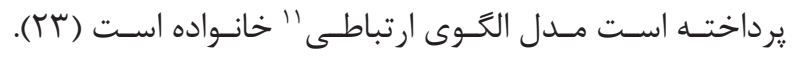

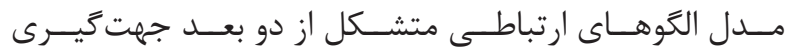

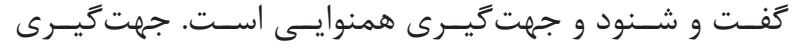

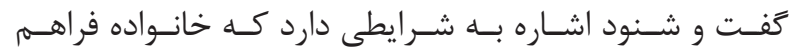

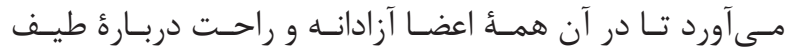

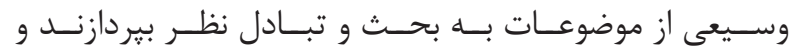

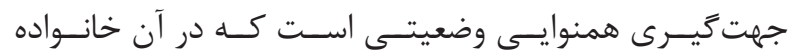

${ }^{1}$ Epilepsy

${ }^{2}$ Alsaadi

${ }^{3}$ Bifftu

${ }^{4}$ Cognitive model

${ }^{5}$ Dysfunctional attitudes

${ }^{6}$ Personality traits

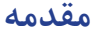

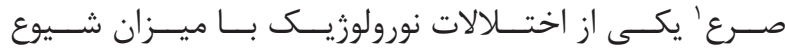

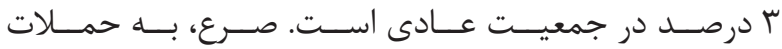

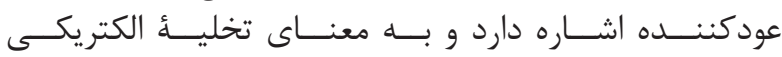

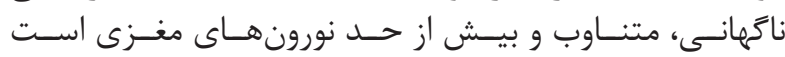

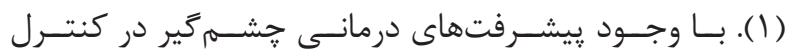

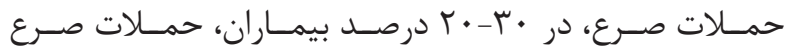

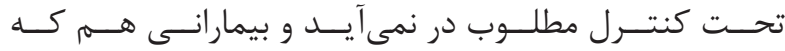

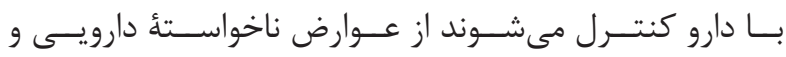

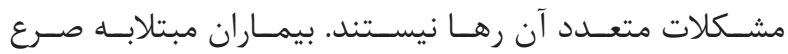

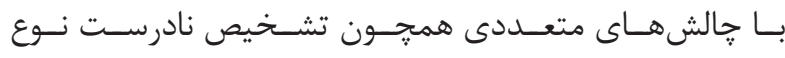

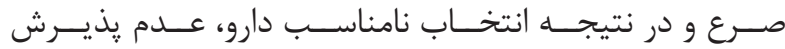

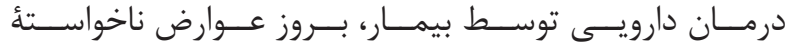

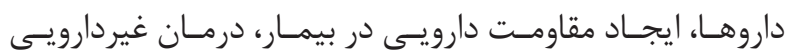

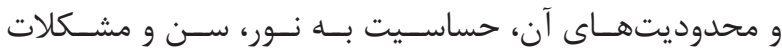

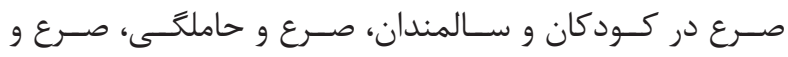

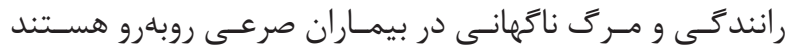

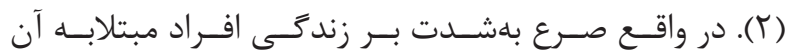

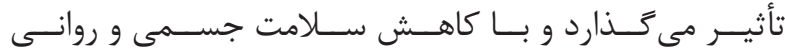

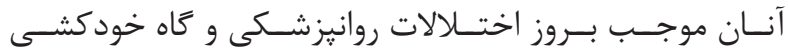

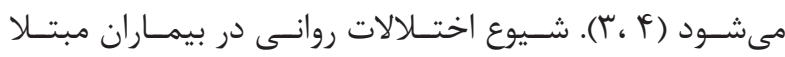

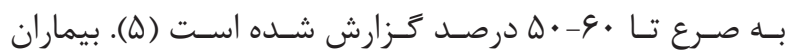

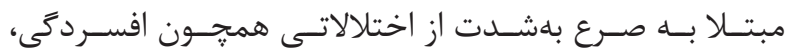

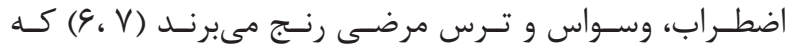

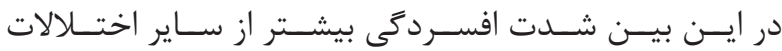

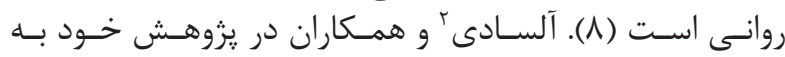

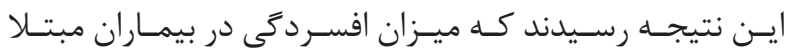

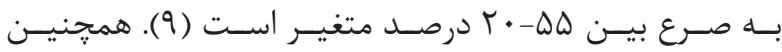

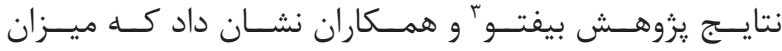

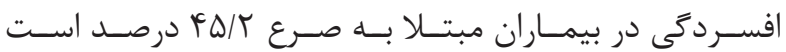

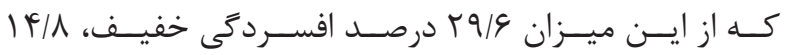

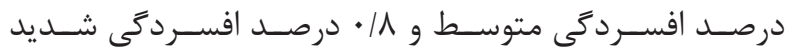

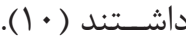

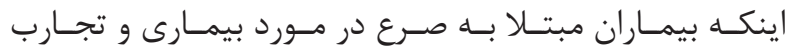

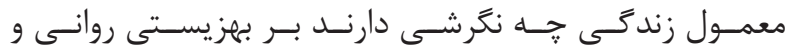

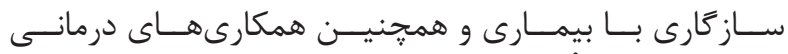

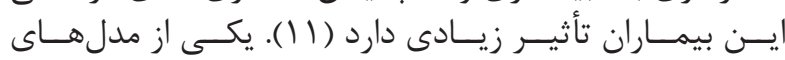

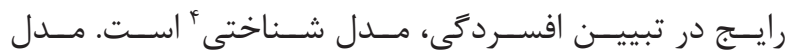

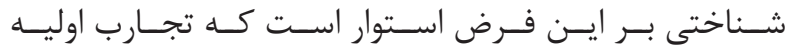

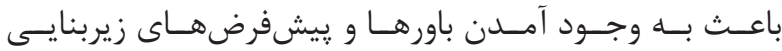

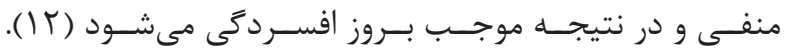

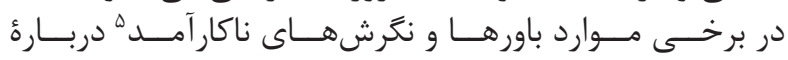

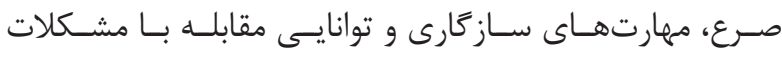

${ }^{7} \mathrm{McCrae}$ and Costa

${ }^{8}$ Harkness

${ }^{9}$ Chioqueta and Stiles

${ }^{10}$ Petersen

${ }^{11}$ Communication patterns 


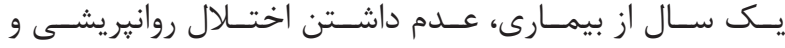

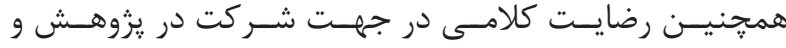

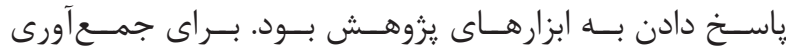

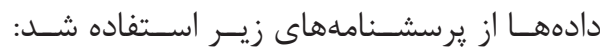

$$
\text { يرسشنامةُ افسردَى بك }
$$

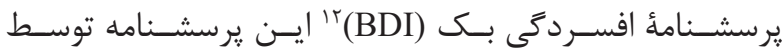

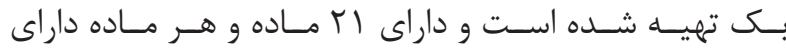

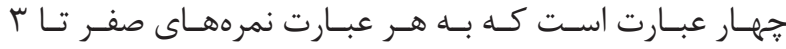

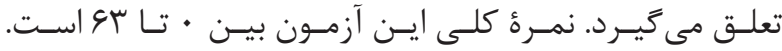

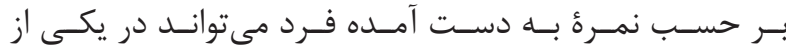

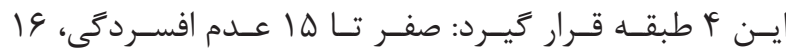

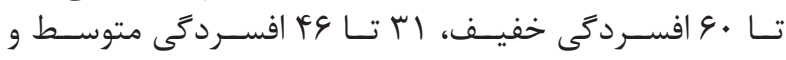

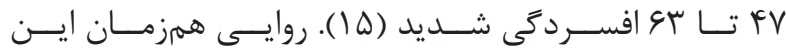

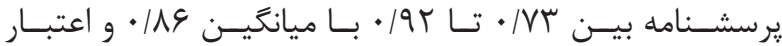

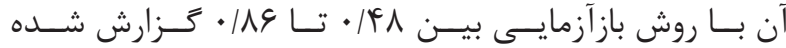

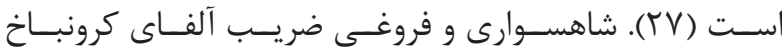

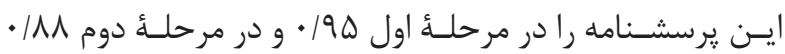

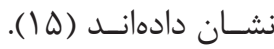

ير سشناملة ينج عاملى نئو

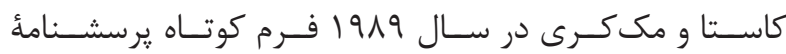

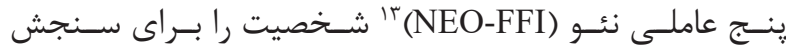

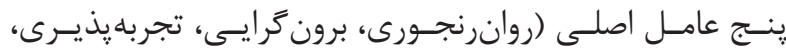

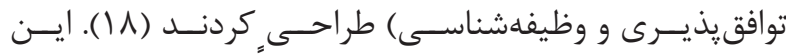

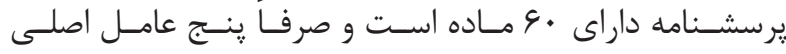

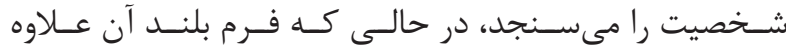

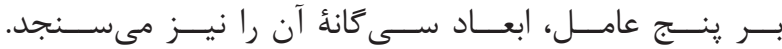

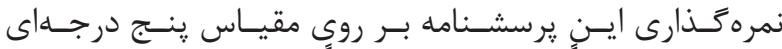

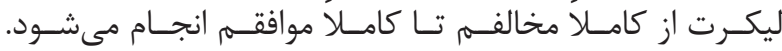

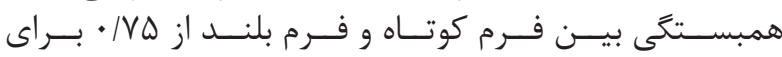

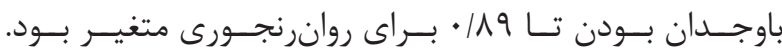

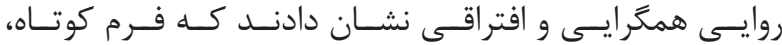

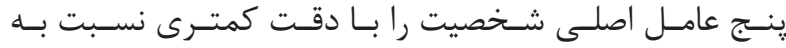

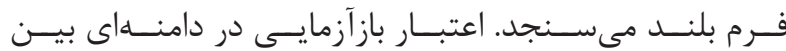

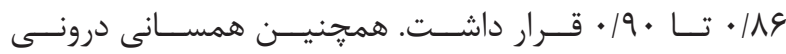

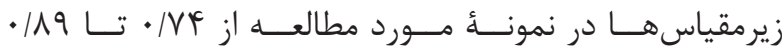

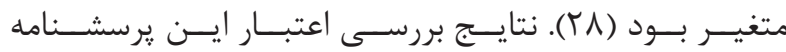

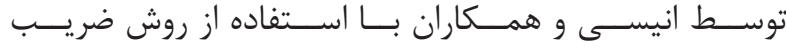

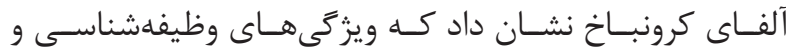

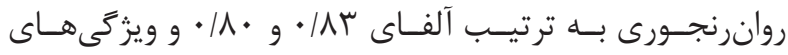

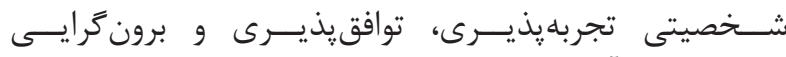

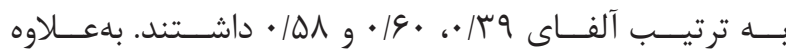

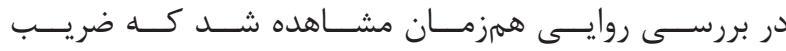

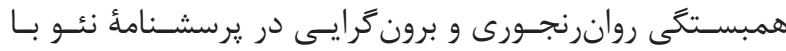

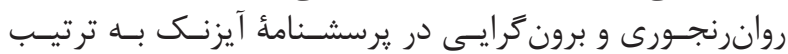

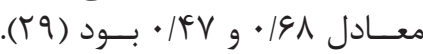

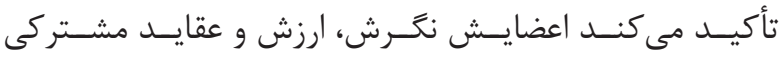

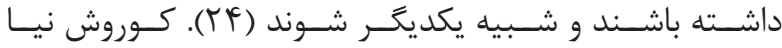

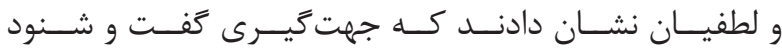

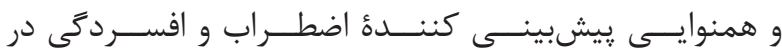
فرزنـدان هســتند (Yه)

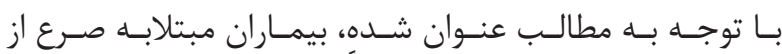

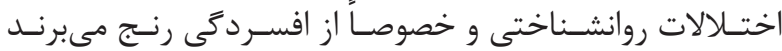

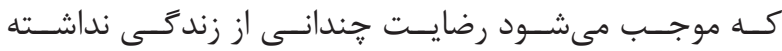

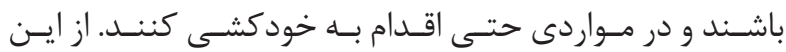

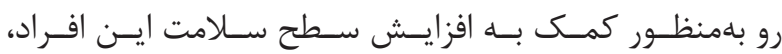

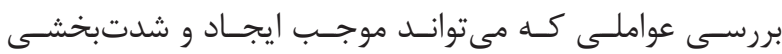

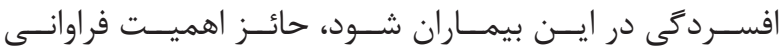

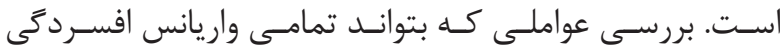

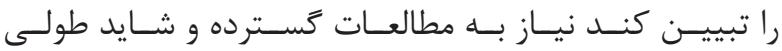

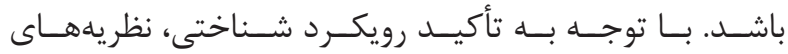

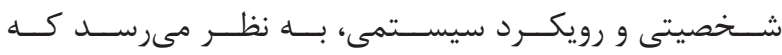

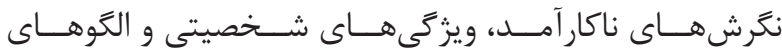

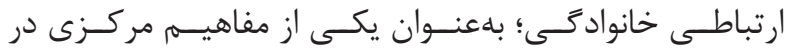

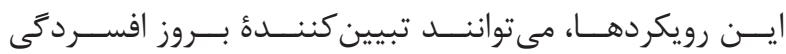

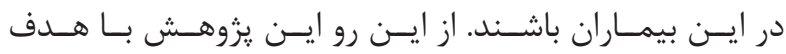

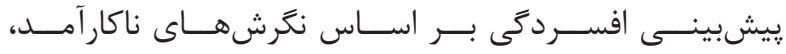

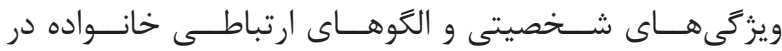

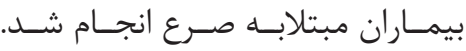

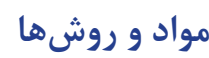

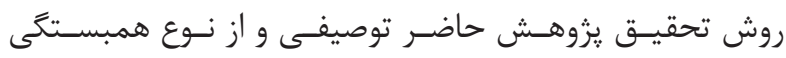

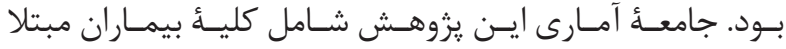

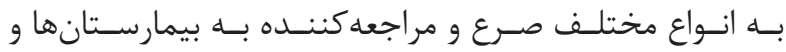

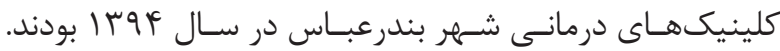

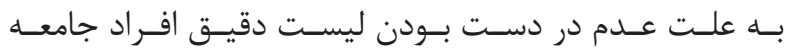

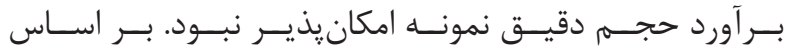

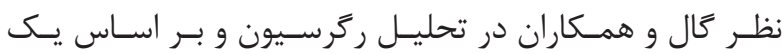

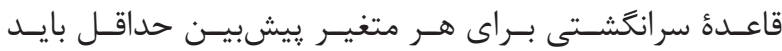

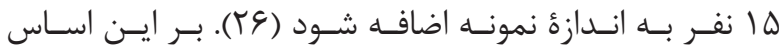

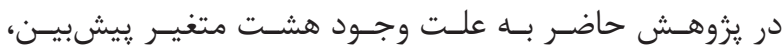

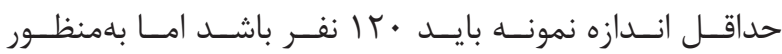

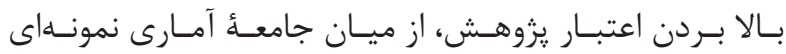

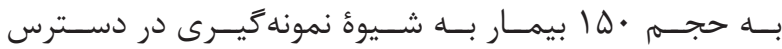

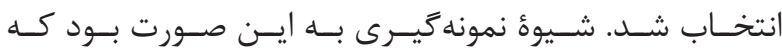

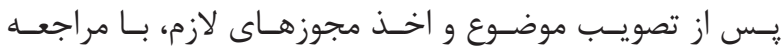

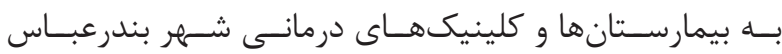

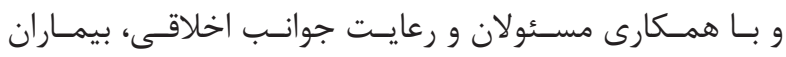

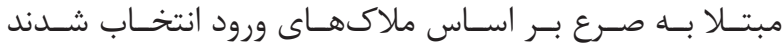

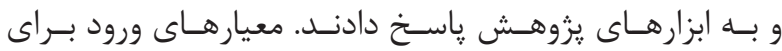

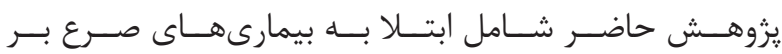

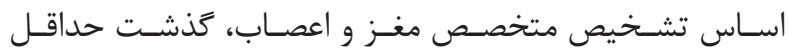




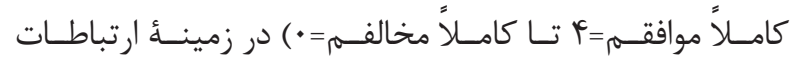

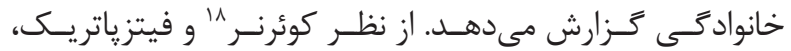

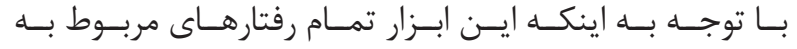

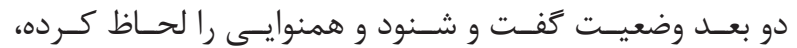

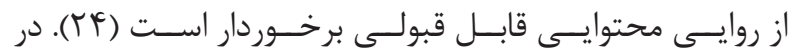

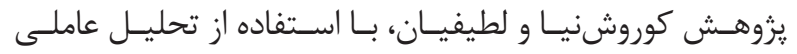

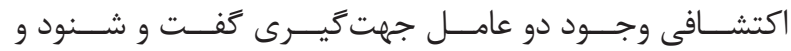

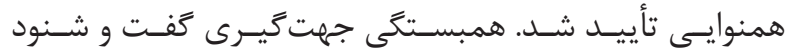

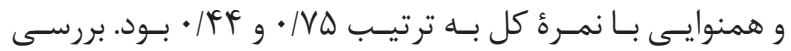

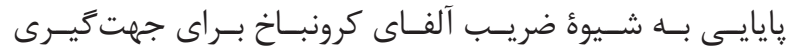

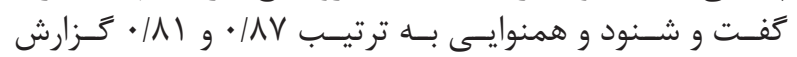

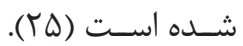

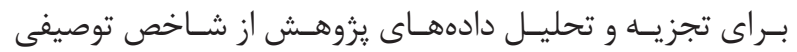

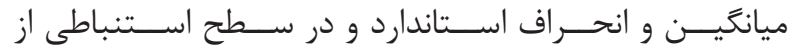

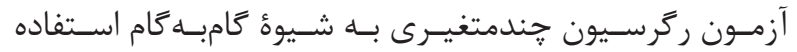

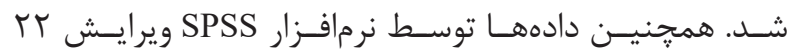

$$
\text { تحليـل شــند همجند. }
$$

بافتهها

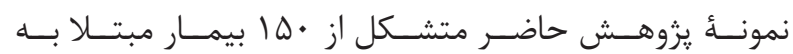

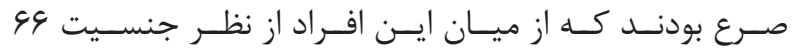

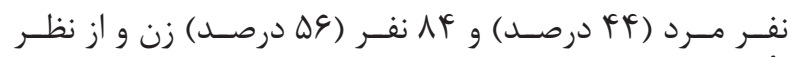

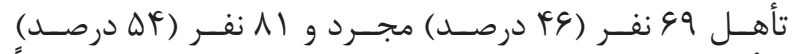

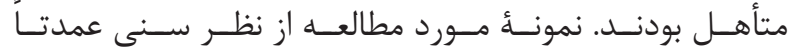

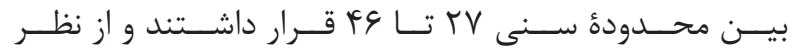

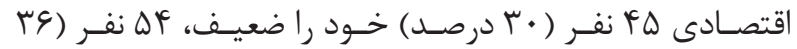

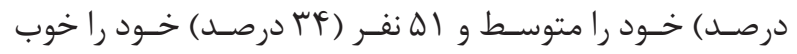

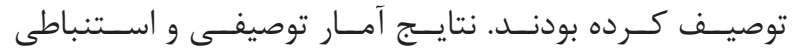

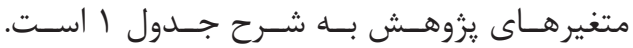

يرسشناملُ نَرش هاى ناكار آمد

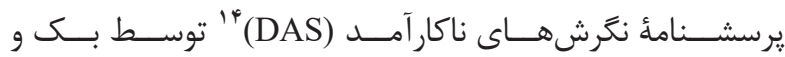

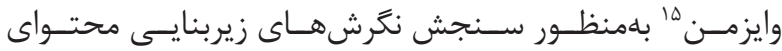

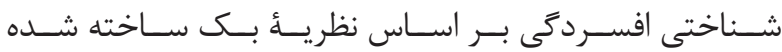

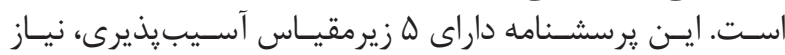

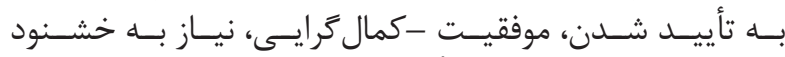

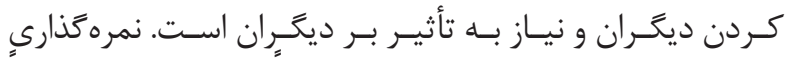

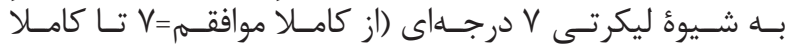

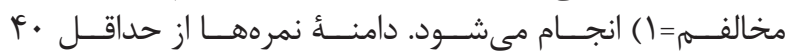

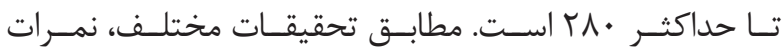

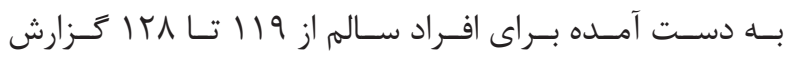

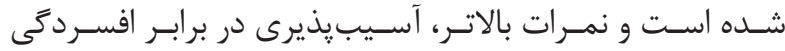

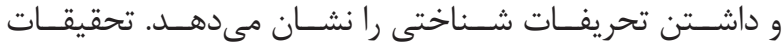

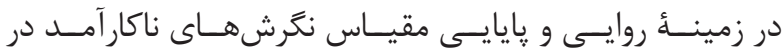

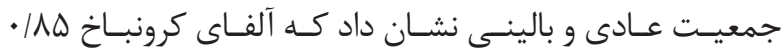

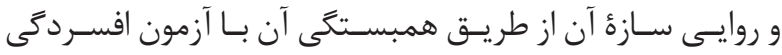

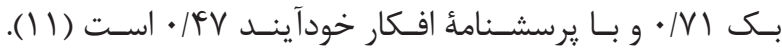

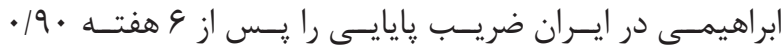

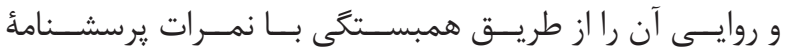

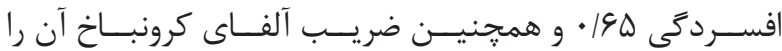

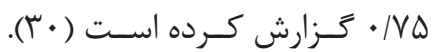
يرسشنامة التَوى ارتباطى خانواده

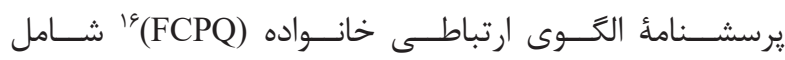

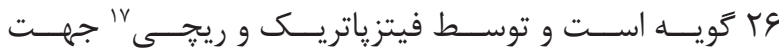

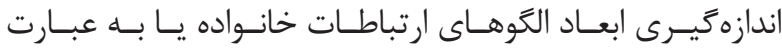

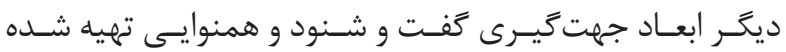

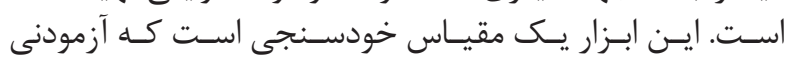

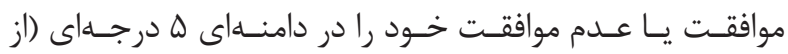

\begin{tabular}{|c|c|c|c|c|c|}
\hline اتحراف معيار & ميانكَين & حداكثر ثمره & حداقل نمره & مؤلفهها & متغيرها \\
\hline$N / F V$ & rN/9 & $\Delta r$ & 19 & برونُمرايى & \multirow{5}{*}{ شخيزئى هاى } \\
\hline F/FT & $+r / V$ & $\Delta f$ & rf & روانرنجورى & \\
\hline$\Delta / \cdot r$ & $f \cdot 1 \cdot 1$ & $\Delta r$ & $r$. & تجربدة ائي & \\
\hline $9(\Delta)$ & $F 1 / 1$ & $\Delta f$ & $r$. & توافقيذيرى & \\
\hline$f /+1$ & $f+/ r$ & $\Delta 1$ & rq & وظيفهشناسى & \\
\hline V/TO & $f \cdot 19$ & VT & 10 & كَت و شنود & \multirow{2}{*}{ الآتوهاى } \\
\hline g/VV & rg/A & Fi & ir & همنوايى & \\
\hline $9 / 1 \wedge$ & TN/F & fr & ir & آسيبديذيرى & \multirow{6}{*}{ ثاكار شهآ آمد } \\
\hline $9 / \mathrm{V}$. & $r \Delta / f$ & fq & $\wedge$ & تأيبد شدن & \\
\hline N/AV & $r \Delta / \Lambda$ & Fq & $\wedge$ & كمالتمرايى & \\
\hline $1 \cdot / 19$ & $r F / \cdot r$ & $\Delta r$ & $\wedge$ & خشنودكردن دينّران & \\
\hline $1 . / \mathrm{VA}$ & rq/1 & $\Delta r$ & $\Lambda$ & تأثير بر ديثُران & \\
\hline rr/f. & $1 r q / 9$ & rrI & 99 & نُكُرشهاى ناكارآ آمد & \\
\hline$r / r v$ & $19 / 4$ & rq & ir & افسردئى & \\
\hline
\end{tabular}

${ }^{14}$ Dysfunctional attitudes scale

${ }^{15}$ Wiseman

${ }^{16}$ Family communication patterns questionnaire
${ }^{17}$ Fitzpatrick and Richie

${ }^{18}$ Koerner 


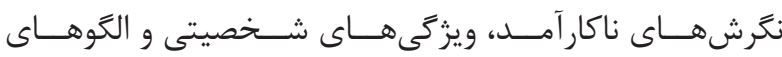

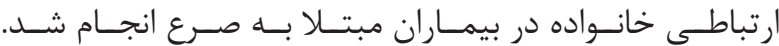

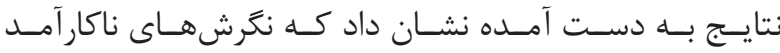

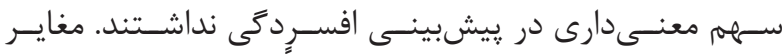

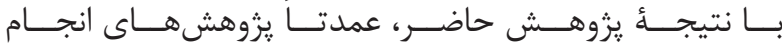

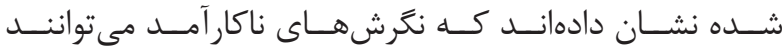

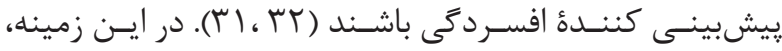

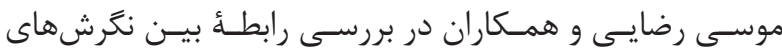

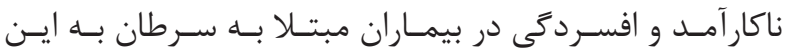

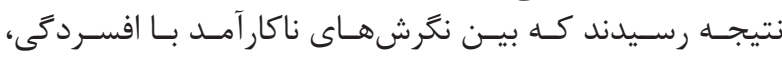

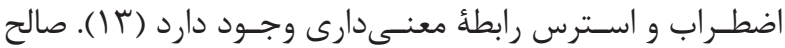

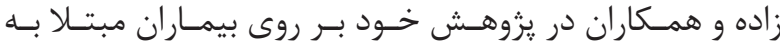

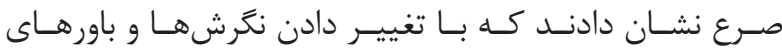

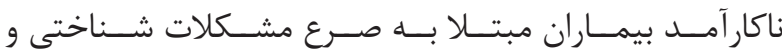

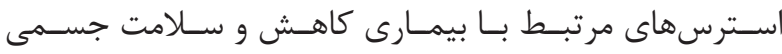

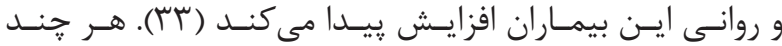

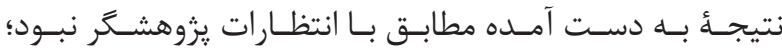

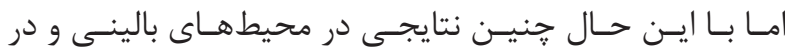

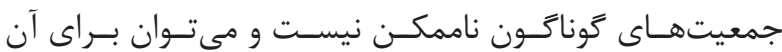

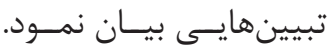

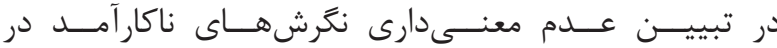

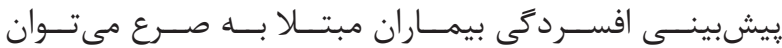

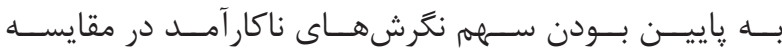

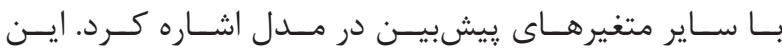

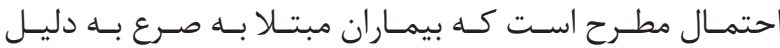

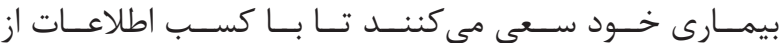

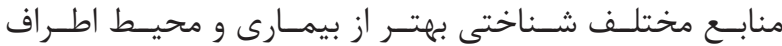

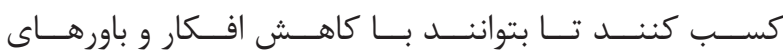

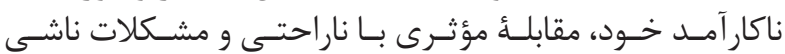

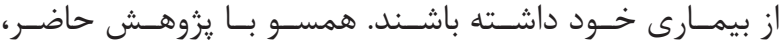

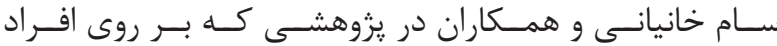

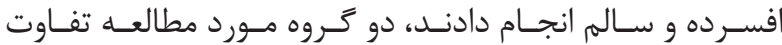

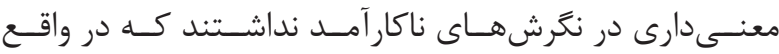

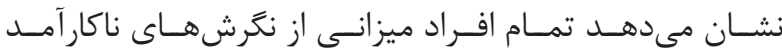

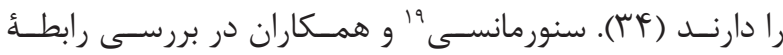

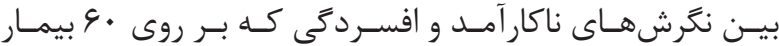

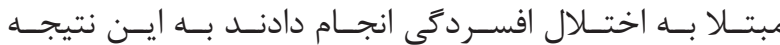

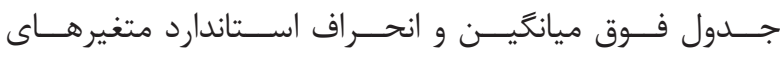

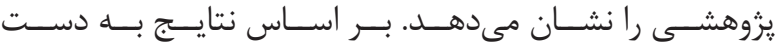

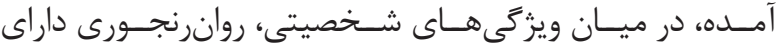
بيشـترين ميانكيـن

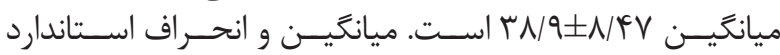

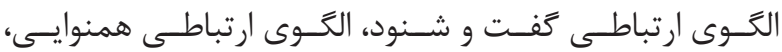

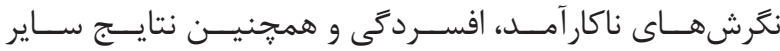

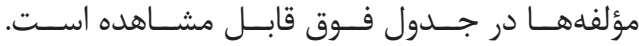

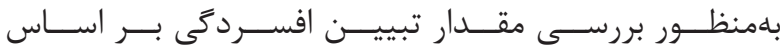

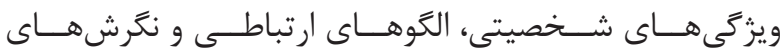

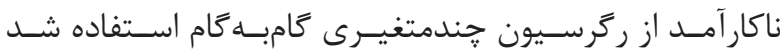

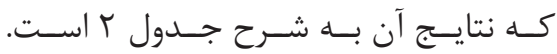

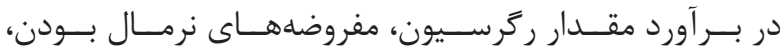

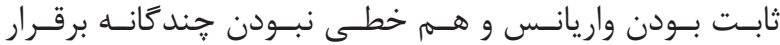

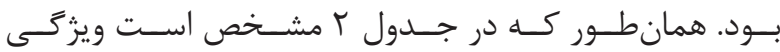

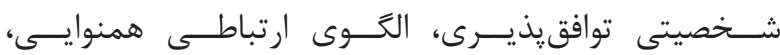

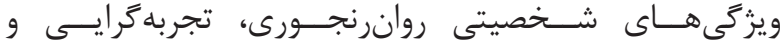

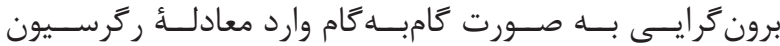

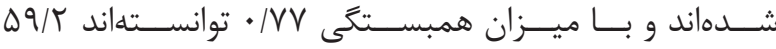

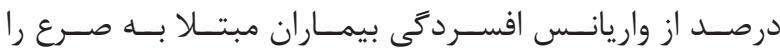

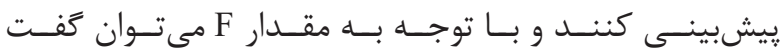

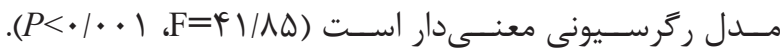

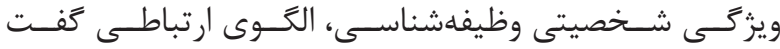

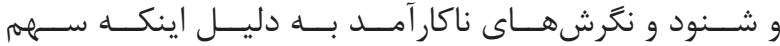

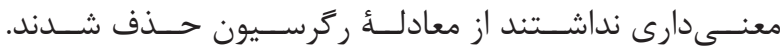

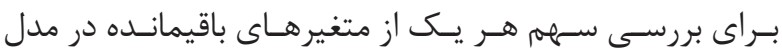

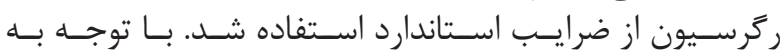

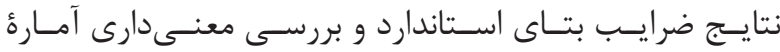

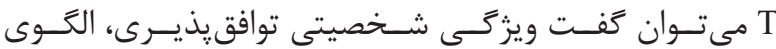

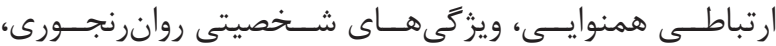

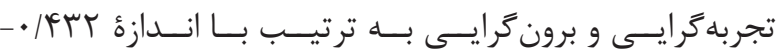
|

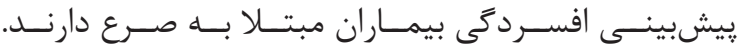
بحث و نتيجه

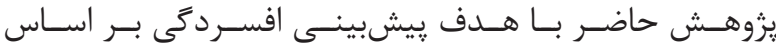

جدول r- نتايج تحليل ركرسيون حندمتغيرى كامبه كام متغيرهاى ييشبين با متغير ملاك افسردىى.

\begin{tabular}{|c|c|c|c|c|c|c|c|c|}
\hline معنى دارى & Tآمارة & خطاى & مقار & 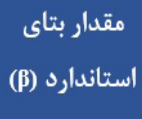 & سطح معنى دارى & ضريب & هندئانه & ميش متغيرهاى \\
\hline.$/ . .1$ & $-9 / N A$ & r & $\cdot \pi I V$ & . & \multirow{5}{*}{$F / / \lambda \Delta$} & \multirow{5}{*}{ - $/ \Delta 9 T$} & \multirow{5}{*}{. /VV } & وافقيذيرى \\
\hline $.1 . .1$ & $F / \Lambda 9$ & .1. YG & ./MT & - RAT & & & & همنوايى \\
\hline $.1 . .1$ & $F / \pi s$ &.$|+4|$ & $\cdot / / \Lambda$. & . & & & & وانرنجورى \\
\hline$\cdot / \cdot r r$ & $-r / 11$ & . I. Fr & $\cdot / / r$. &.$- / 199$ & & & & نجربهكرايى \\
\hline$\cdot / \cdot 1$ & $-r / N$. &.$/ . T Y$ & .1 .9$. & $\cdot / / 1 \Delta \Delta$ & & & & برون كرايى \\
\hline
\end{tabular}




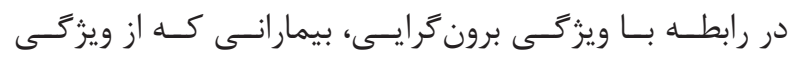

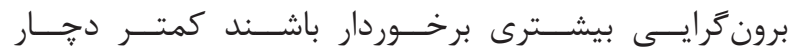

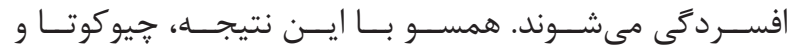

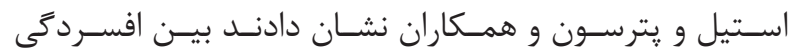

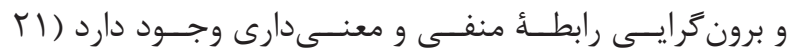

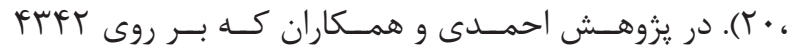

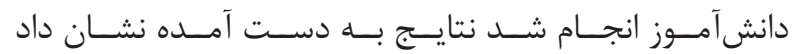

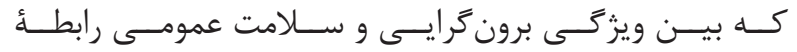

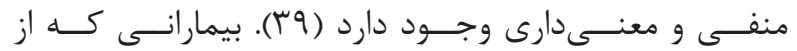

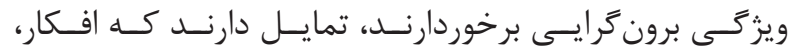

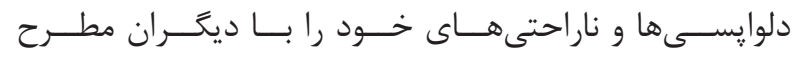

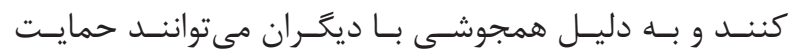

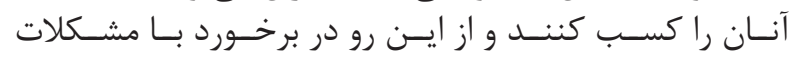

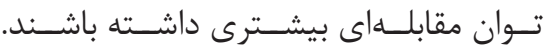

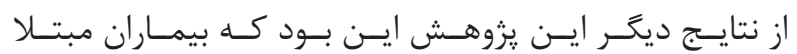

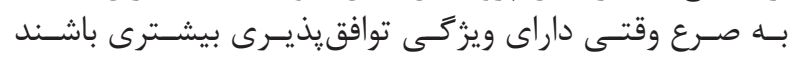

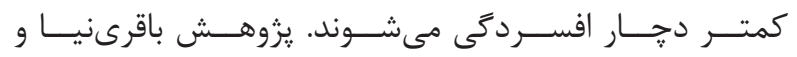

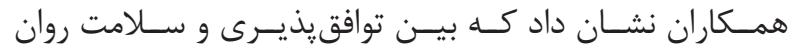

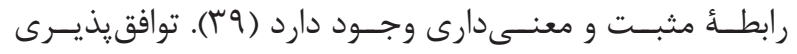

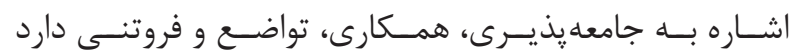

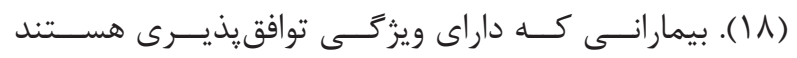

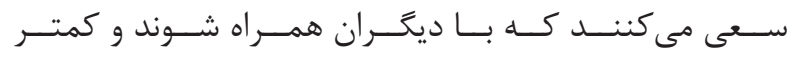

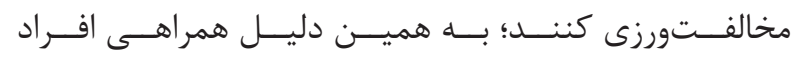

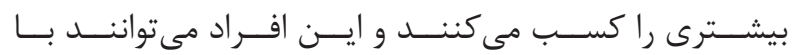

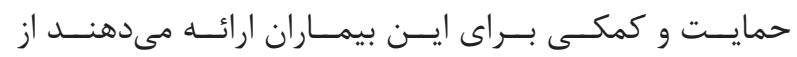

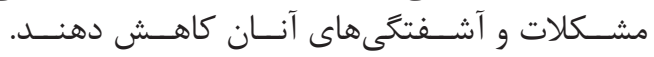

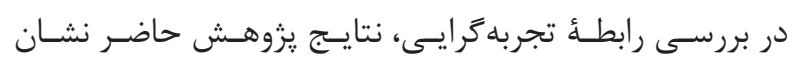

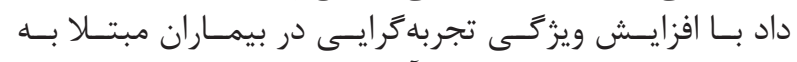

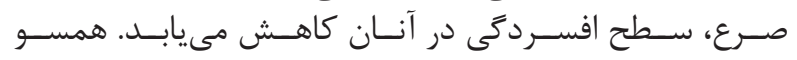

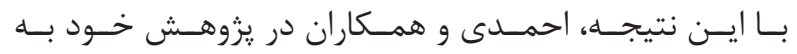

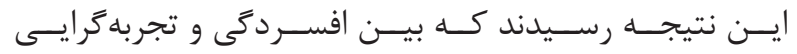

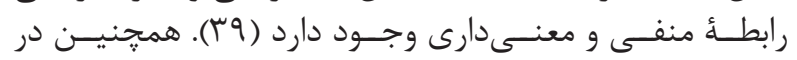

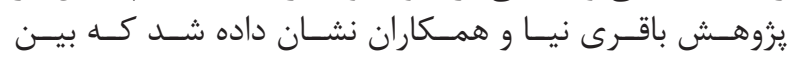

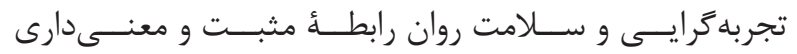

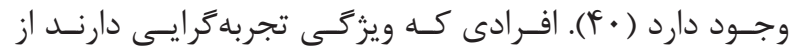

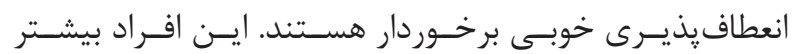

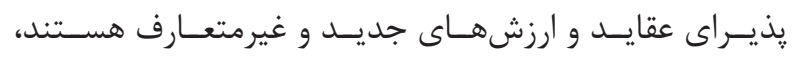

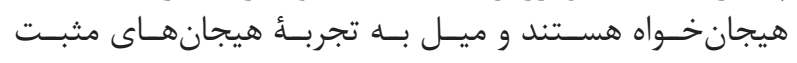

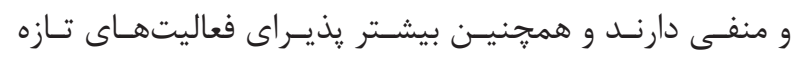

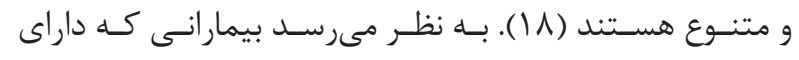

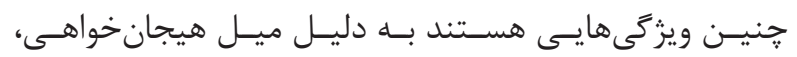

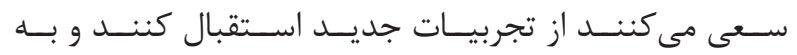

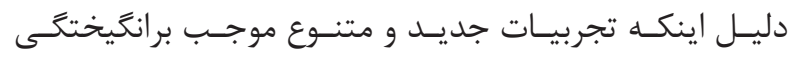

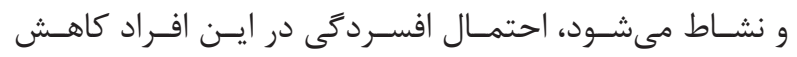
ييــدا مى كنـــد.

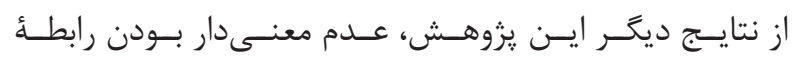

${ }^{20}$ Kotov

${ }^{21}$ Mak

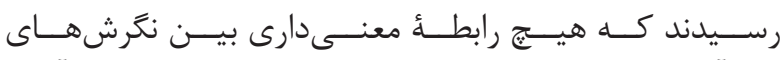

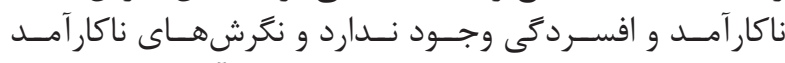

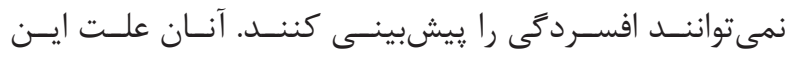

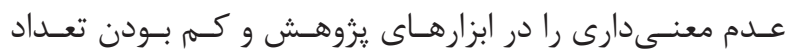

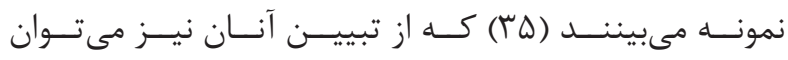

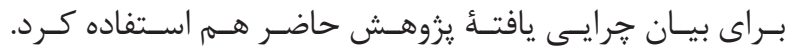

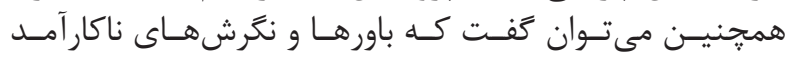

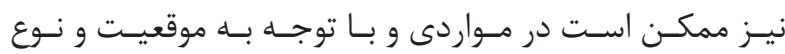

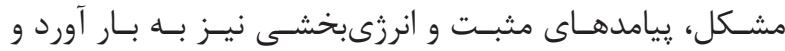

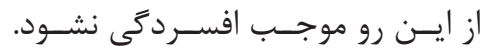

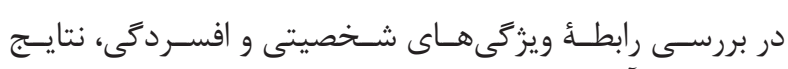

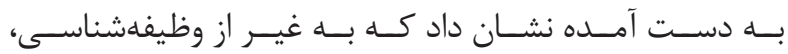

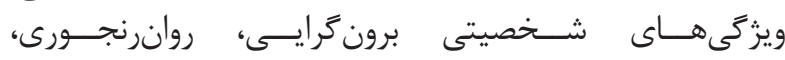

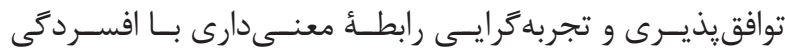

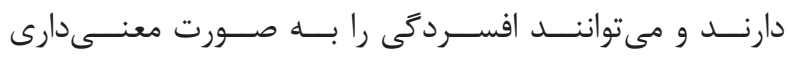

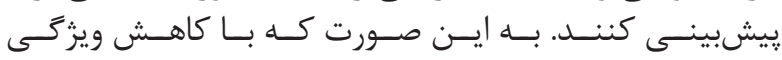

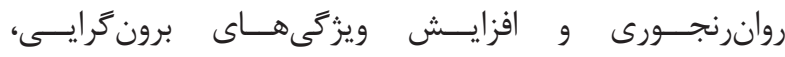

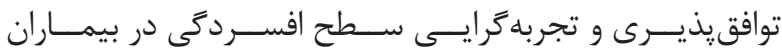

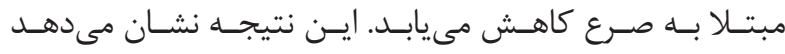

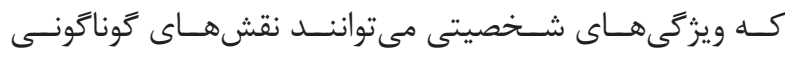

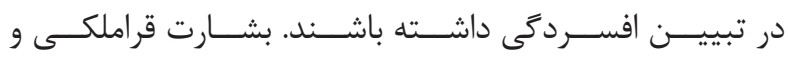

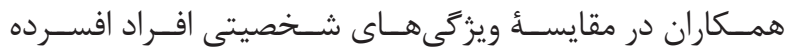

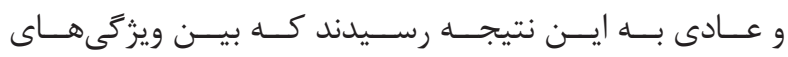

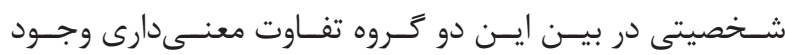

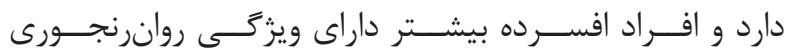

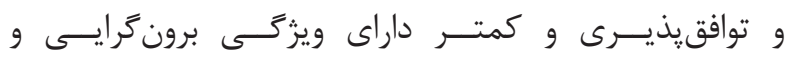

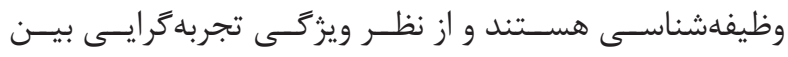

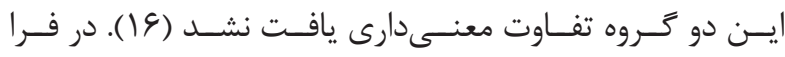

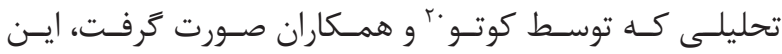

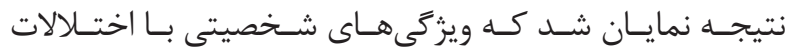

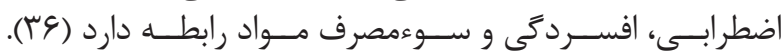

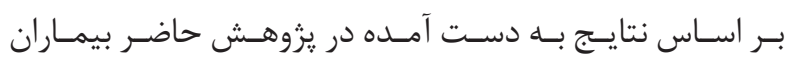

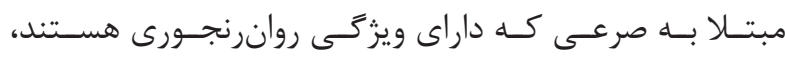

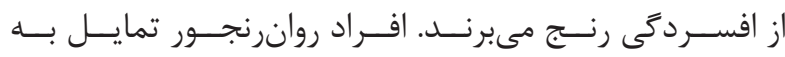

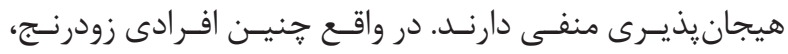

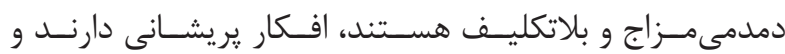

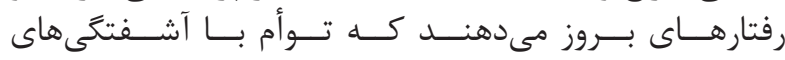

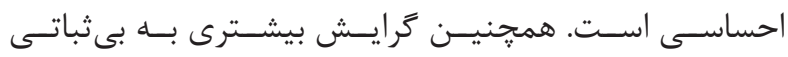

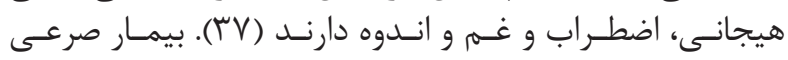

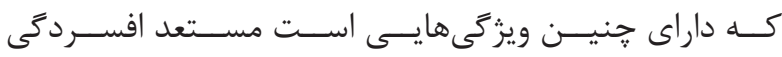

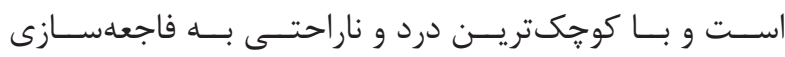

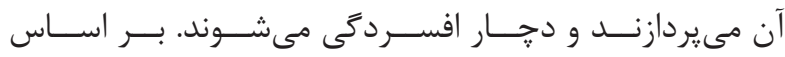

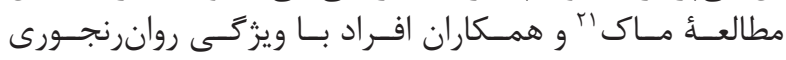

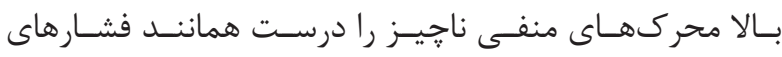

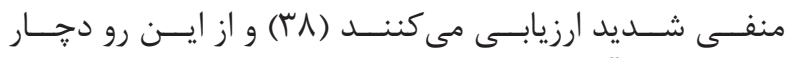

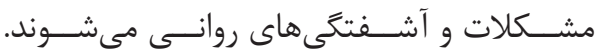




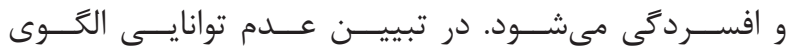

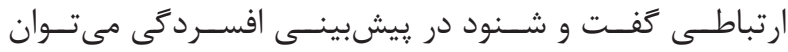

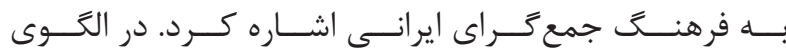

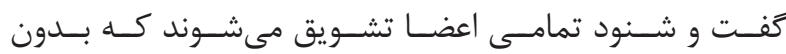

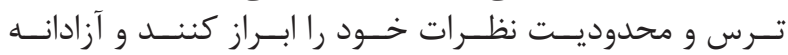

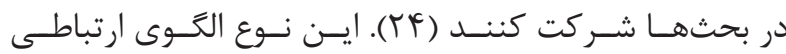

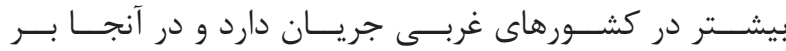

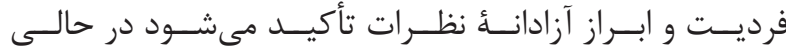

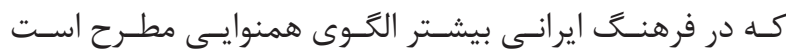

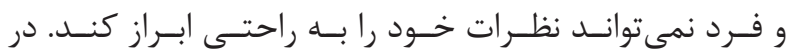

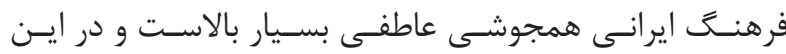

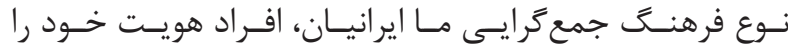

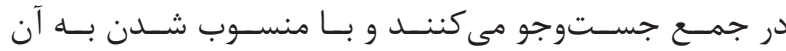

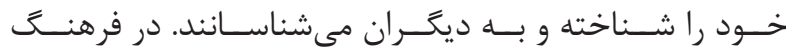

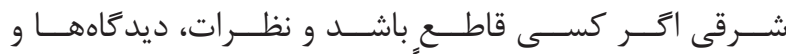

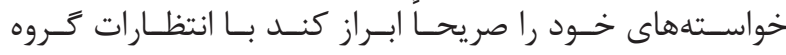

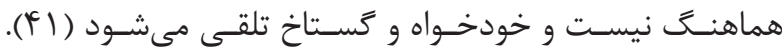

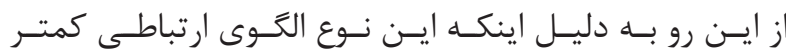

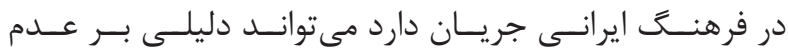

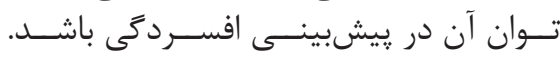

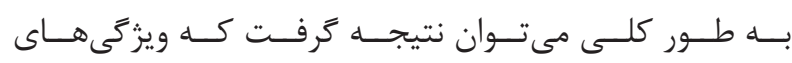

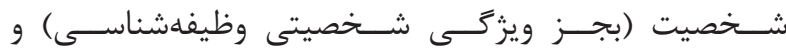

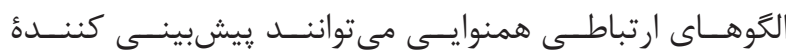

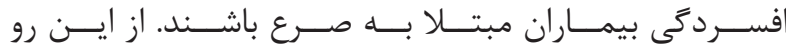

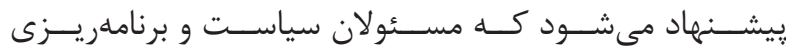

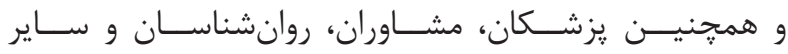

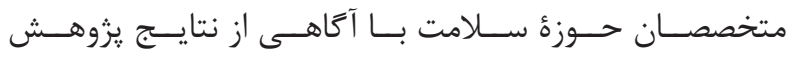

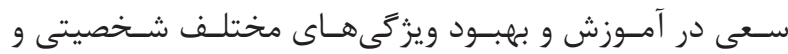

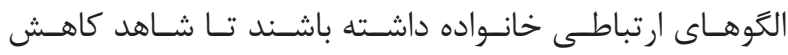

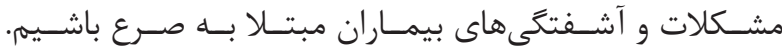

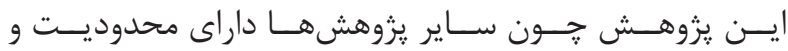

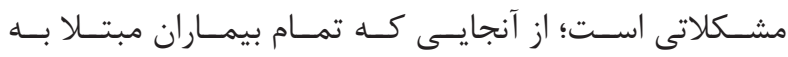

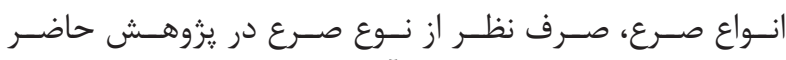

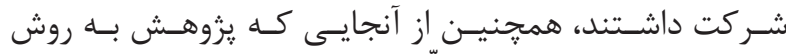

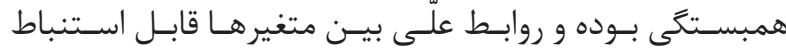

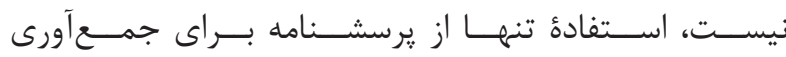

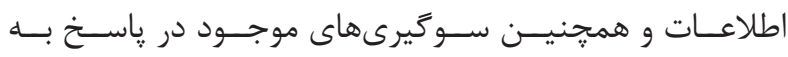

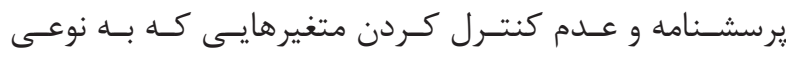

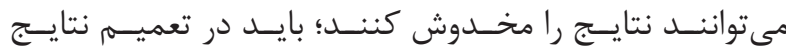

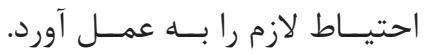

$$
\text { تشكر و قدردانى }
$$

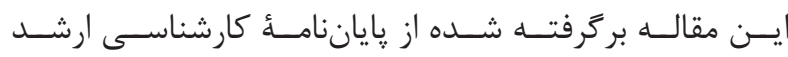

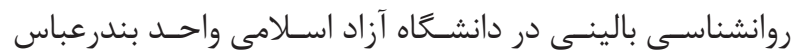

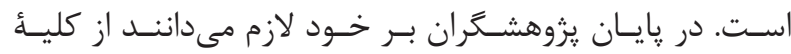

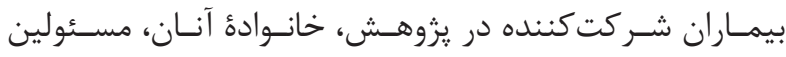

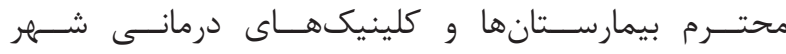

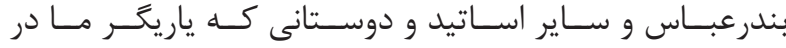

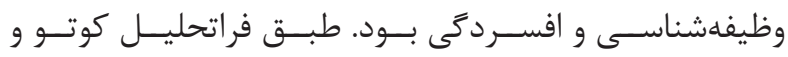

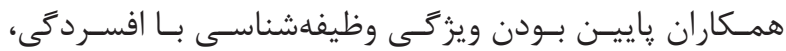

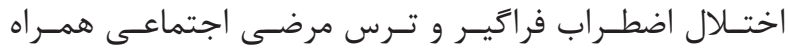

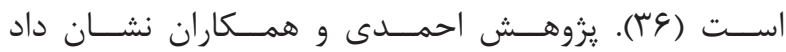

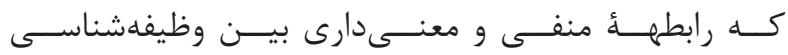

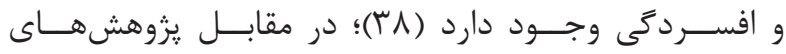

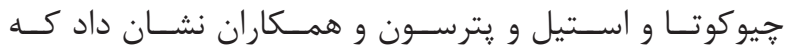

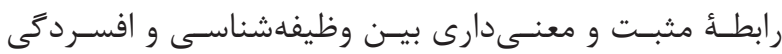

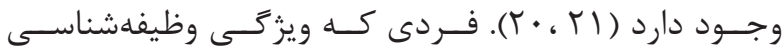

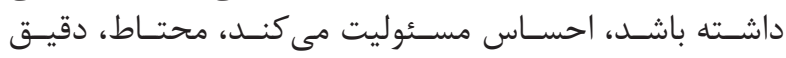

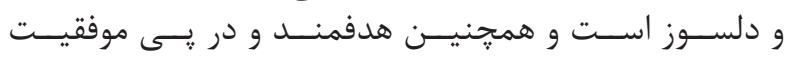

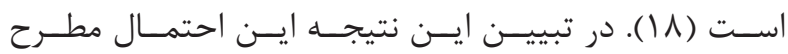

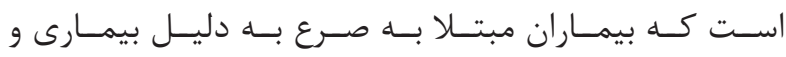

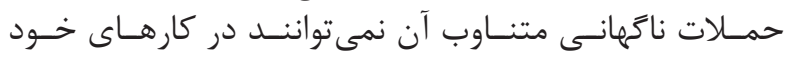

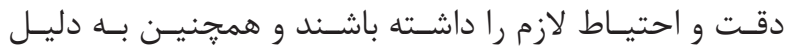

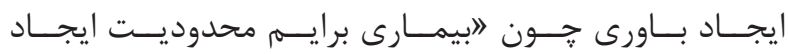

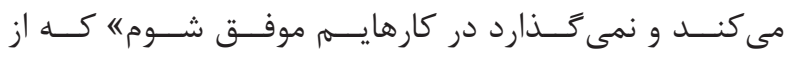

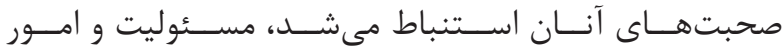

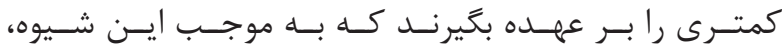

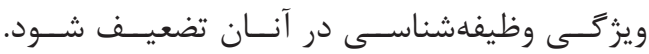

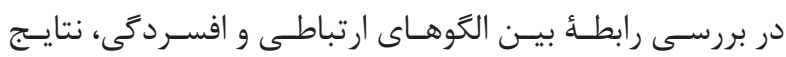

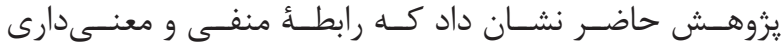

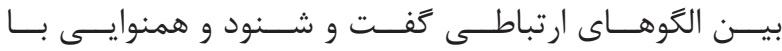

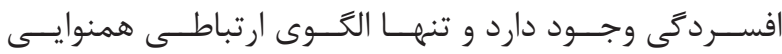

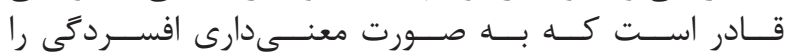

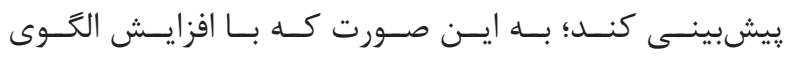

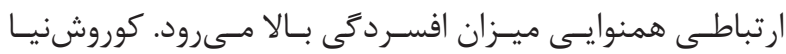

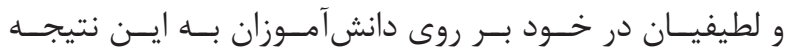

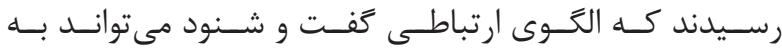

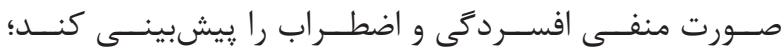

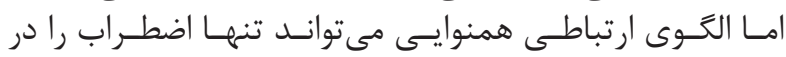

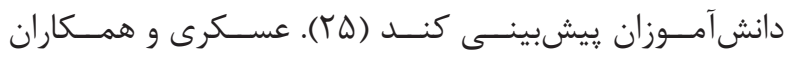

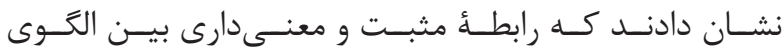

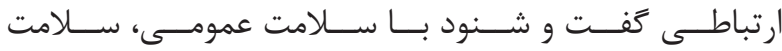

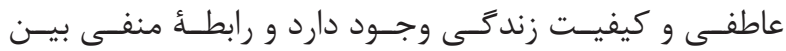

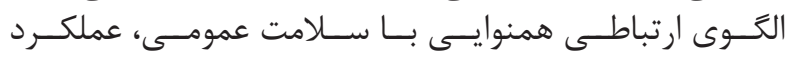

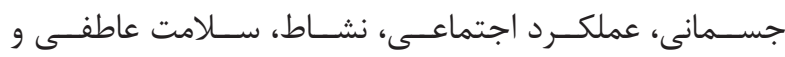

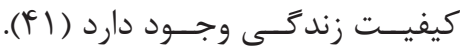

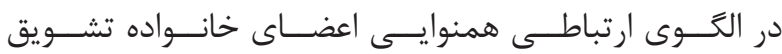

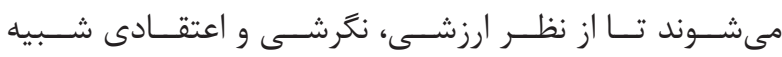

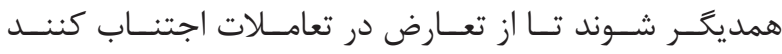

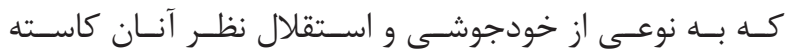

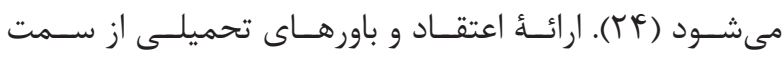

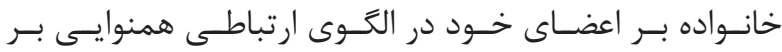

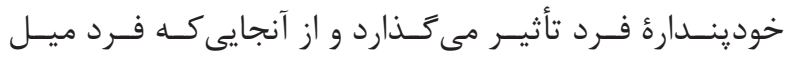

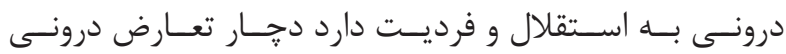

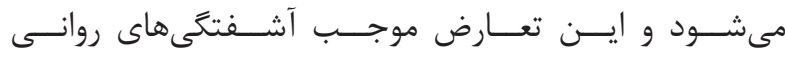


ايسن يزووهـش بودهانــد صميمانــه تشـكر و قدردانسى نماينــد. 1. Najafi MR, Rezaei F, Vakili Zarch N, Dehghani F, Barekatain M. Personality and psychopathology of patients with grandmal and complex partial seizures. J Shahid Sadoughi Univ Med Sci. 2010; 18(2): 84-91.

2. Ebrahimi HA. Challenges in the treatment of epilepsy. J Kerman Univ Med Sci. 2012; 19(2): 212-24.

3. Kerr MP. The impact of epilepsy on patients' lives. Acta Neurol Scand. 2012; 126(194): 1-9.

4. Hesdorffer DC, Ishihara L, Mynepalli L, Webb DJ, Weil J, Hauser WA. Epilepsy, suicidality, and psychiatric disorders: a bidirectional association. Ann Neurol. 2012; 72(2): 184-91.

5. Dehghanifiroozabadi M, Manshaee G, Danae Sij Z, Sharifzadeh G. Effectiveness of cognitive behavioral stress management on depression and anxiety symptoms of patients with epilepsy and migraine. J Birjand Univ Med Sci. 2015; 21(4): 407-15.

6. Kwon OY, Park SP. Depression and anxiety in people with epilepsy. J Clin Neurol. 2014; 10(3): 175-88.

7. Noohi S, Azar M, Karamad A, Shafiee Kandjani A, Amiri M, Habibi M et al. Frequency of symptoms of depression, general anxiety, obsessive- compulsive and phobia in interictal phase in Patients with epilepsy. Med Sci. 2008; 18(1): 39-43.

8. Gandy M, Sharpe L, Perry KN. Psychosocial predictors of depression and anxiety in patients with epilepsy: a systematic review. J Affect Disord. 2012; 140(3): 222-32.

9. Alsaadi T, El Hammasi K, Shahrour TM, Shakra M, Turkawi L, Nasreddine W, et al. Depression and anxiety among patients with epilepsy and multiple sclerosis: UAE comparative study. Behavioural Neurology. 2015; 4. doi.org/10.1155/2015/196373.

10. Bifftu BB, Dachew BA, Tiruneh BT, Tebeje NB. Depression among people with epilepsy in Northwest Ethiopia: a cross-sectional institution based study. BMC Research Notes. 2015; 8(585): 1-8.

11. Salehzadeh M, Najafi M. Effectiveness of cognitivebehavioral therapy on dysfunctional attitudes in epileptic patients. J Shahid Sadoughi Univ Med Sci. 2011; 19(3): 377-87.

12. Salehzadeh M, kalantari M, molavi H, Najafi MR, Ebrahimi A. Effectiveness of cognitive-behavioral group therapy on depression in intractable epileptic patients. Adv Cog Sci. 2012; 12(2): 59-68.

13. Musarezaie A, Khaledi F, Kabbazi-fard M, Momeni-GhaleGhasemi T, Keshavarz M, Khodaee M. Investigation the dysfunctional attitudes and its' relationship with stress, anxiety and depression in breast cancer patients. J Health Syst Res. 2015; 11(1): 68-76.

14. Ebrahimi A, Neshatdoost H, Kalantari M, Molavi $\mathrm{H}$, Asadollahi G. Contributions of dysfunctional attitude scale and general health subscales to prediction and odds ratio of depression. J Shahrekord Univ Med Sci. 2008; 9(4): 52-8.

15. Shahsavari A, Foroghi S. Effectiveness of cognitive therapy on depression in epileptic patients. IJPN. 2015; 3(1): 37-46.

16. Besharat Gharamaleki R, Khanjani Z, Babapour J. Comparison of five big personality factors in depressive disorder patients and obsessive -compulsive disorders with normal individuals. Knowl Res Appl Psycho. 2013; 14(1): 110-7.

17. Lawrence AP, Oliver PJ. Handbook of personality: theory and research. $3^{\text {rd }}$ ed. New York: Guilford; 1989.

18. McCrae RR, Costa PT Jr. Rotation to maximize the construct validity of factors in the NEO personality inventory. Multivariate Behav Res. 1989; 24(1): 107-24.

19. Harkness KL, Michael Bagby R, Joffe RT, Levitt A. Major depression, chronic minor depression, and the five-factor model of personality. Eur J Pers. 2002; 16(4): 271-81.

20. Chioqueta AP, Stiles TC. Personality traits and the development of depression, hopelessness, and suicide ideation. Pers Individ Dif. 2005; 38(6): 1283-91.

21. Petersen T, Papakostas GI, Bottonari K, Iacoviello B, Alpert JE, Fava M, et al. NEO-FFI factor scores as predictors of clinical response to fluoxetine in depressed outpatients. Psychiatry Res. 2002; 109(1): 9-16.

22. Radfar M, Ahmadi F, Fallahi Khoshknab M. Depression roots in viewpoints of depressed patients' family. J Urmia Nurs Midwifery Fac. 2013; 10(6): 78092.

23. Fitzpatrick MA. Family communication patterns theory: Observations on its development and application. J Fam Commun. 2004; 4(3-4): 167-79. 


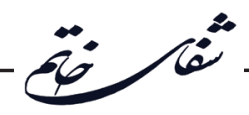

24. Koerner AF, Fitzpatrick MA. Family type and conflict: The impact of conversation orientation and conformity orientation on conflict in the family. Commun Stud. 1997; 48(1): 59-75.

25. Kouroshnia M, latifian M. Relationship between family communication patterns with anxiety and depression in children. J Fam Res. 2007; 3(10): 587-600.

26. Gall MD, Borg WR, Gall JP. Educational research: an introduction. Longman Publishing; 1996.

27. Zahiroddin A, Ghoreishi F. A survey of depressive symptoms in patients. Res in Med. 2006; 30(4): 305-8.

28. Roshanchsly R, Shaeiri M, Atryfrd M, Nikkhah A, Qaem-Maqami B, Rahimi-Rad A. Psychometric properties of "five-factor NEO personality inventory NEO-FFI. J Daneshvar-Raftar. 2007; 16: 27-36.

29. Anisi J, Majdiyan M, Joshanloo M, Ghoharikamel Z. Validity and reliability of NEO five-factor inventory (NEO-FFI) on university students. J Behav Sci. 2011; 5(4): $17-8$.

30. Ebrahimi A. Effectiveness of integrated treatment of religious, cognitive behavioral and drug therapy on depression and dysfunctional attitudes of patients with dysthymic disorder. $\mathrm{PhD}$ thesis. Isfahan University; 2009.

31. Moallemi S, Bakhshani NM, Raghibi M. On the relationship between mental health, spiritual intelligence and dysfunctional attitudes in students of Systan and Baluchestan University, Southeast of Iran. J Fund Ment Health. 2011; 12(4): 702-9.

32. Shankman SA, Campbell ML, Klein DN, Leon AC, Arnow BA, Manber R, et al. Dysfunctional attitudes as a moderator of pharmacotherapy and psychotherapy for chronic depression. J Psychiatric Res. 2013; 47(1): 113-21.

33. Salehzade M, Kalantari M, Molavi H, Najafi MR,
Noori A. Effectiveness of cognitive - behavioral group therapy with focusing on dysfunctional attitudes in epilepsy on quality of life in intractable epileptic patients. J Behav Sci. 2011; 4(4): 255-60.

34. Samkhaniani N, Yazdandoost R, Asgharnejad Farid AA. Surveying schematic mental model, perfectionism and need for approval, in depression. IJPCP. 2003; 8(3): 65-74.

35. Senormanci O, Konkan R, Guclu O, Senormanci G, Sungur MZ. The relationship between dysfunctional attitudes, rumination response styles and depression. Dusunen Adam. 2013; 26(3):239-47.

36. Kotov R, Gamez W, Schmidt F, Watson D. Linking "big" personality traits to anxiety, depressive, and substance use disorders: a meta-analysis. Psychol Bull. 2010; 136(5): 768-821.

37. Handelzalts JE, Becker G, Ahren MP, Lurie S, Raz N, Tamir Z, Sadan O. Personality, fear of childbirth and birth outcomes in nulliparous women. Arch Gynecol Obstet. 2015; 291(5): 1055-62.

38. Mak AS, Blewitt K, Heaven PC. Gender and personality influences in adolescent threat and challenge appraisals and depressive symptoms. Pers Individ Dif. 2004; 36(6): 1483-96.

39. Ahmadi K, Nejati V, Abdi M. Comparison of general health in adolescent boys and girls and its relationship with personality characteristics. J Guilan Univ Med Sci. 2012; 21(81): 29-36.

40. Bagherinia H, Yamini M, Ildarabadi S, Bagherinia F. Relationship between personality traits and mental health with resilience mediation in nurses. J Sabzevar Univ Med Sci. 2016; 22(6): 1063-70.

41. Askari S, Rashidi AR, Naghshineh T, Sharifi S. Relationship between family communication patterns and self-differentiation with quality of life in Seekingdivorce women. J Rooyesh-e-Ravanshenasi. 2015; 4(11): 87-112. 